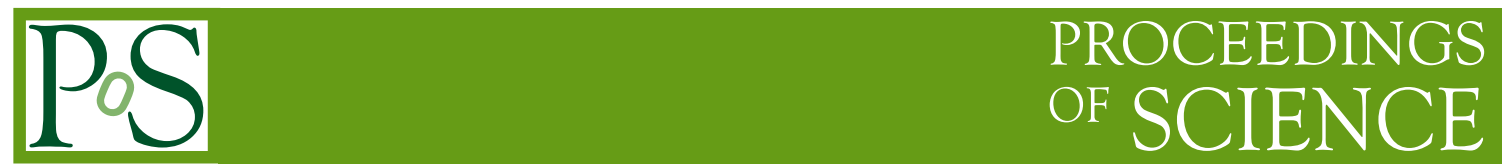

\title{
Extensions in FormCalc 5.3
}

\section{T. Hahn*}

Max-Planck-Institut für Physik,

Föhringer Ring 6, D-80805 Munich, Germany

\section{J.I. Illana}

Departamento de Física Teórica y del Cosmos, and Centro Andaluz de Física de Partículas Elementales (CAFPE),

Universidad de Granada,

E-18071 Granada, Spain

We present a new tool for editing Feynman diagrams as well as several extensions in version 5.3 of the package FormCalc for the calculation of Feynman diagrams.

XI International Workshop on Advanced Computing and Analysis Techniques in Physics Research April 23-27 2007

Amsterdam, the Netherlands

*Speaker. 


\section{Introduction}

FeynArts [1] and FormCalc [2] are Mathematica packages for the generation and calculation of Feynman diagrams up to one-loop order. This contribution describes a new tool for graphically editing Feynman diagrams in FeynArts' $\mathrm{LT}_{\mathrm{E}} \mathrm{X}$ format as well as several new features of FormCalc:

- A Mathematica interface for FormCalc-generated Fortran code.

- The splitting of abbreviations into tree and loop parts (this can greatly affect performance).

- The implementation of four-dimensional Fierz identities.

- A new function to express amplitudes in terms of phase-space variables fully analytically.

- New functions for easier definition of renormalization constants.

- A separate diagonalization package.

\section{Editing Feynman Diagrams}

FeynEdit is a Java program for editing Feynman diagrams. It uses the $\mathrm{LT}_{\mathrm{E}} \mathrm{X}$ representation of FeynArts [1] for input and output. Diagrams are entered into and retrieved from the editor through cut-and-paste with the mouse. This makes it unnecessary to first save the diagrams one wants to edit in a separate file.

Changing the geometry of a diagram has thus become easy. At least in the present version, however, the editor does not show details such as line styles and the actual labels. This is mostly for performance reasons (think of dragging a gluon line). With the FeynArts $\mathrm{LT}_{\mathrm{E}} \mathrm{X}$ format, it is not difficult to change these elements later in a text editor.

The window is divided into an upper panel for the diagram display and a lower panel which shows the $\mathrm{LT}_{\mathrm{E}} \mathrm{X}$ code (see Fig. 1). To display an existing Feynman diagram, mark its LATEX code with the mouse and paste it into the lower dialog box. Then press the 岀 button to display the diagram. Otherwise, start with an empty canvas and use the mouse to add elements.

When finished with editing, press the $\pi$ button to turn the diagrams into LTEX code, then pick up the latter with the mouse and paste it (back) into your text.

The diagram can be edited with the mouse. Four editing functions are available:

- Move vertices, propagators, and labels: Click on the corresponding box (red, blue, green) and drag it to the desired position.

- Draw tadpoles: Click on the 'footpoint' of the tadpole and drag it to the desired size and orientation.

- Draw 'ordinary' propagators: Click on the starting point and drag to the end point.

- Delete objects: Click on the square (red, blue, green) corresponding to the object you want to delete. When deleting a vertex, the propagators adjacent to this vertex are also deleted. When deleting a propagator, the corresponding label is also deleted. 

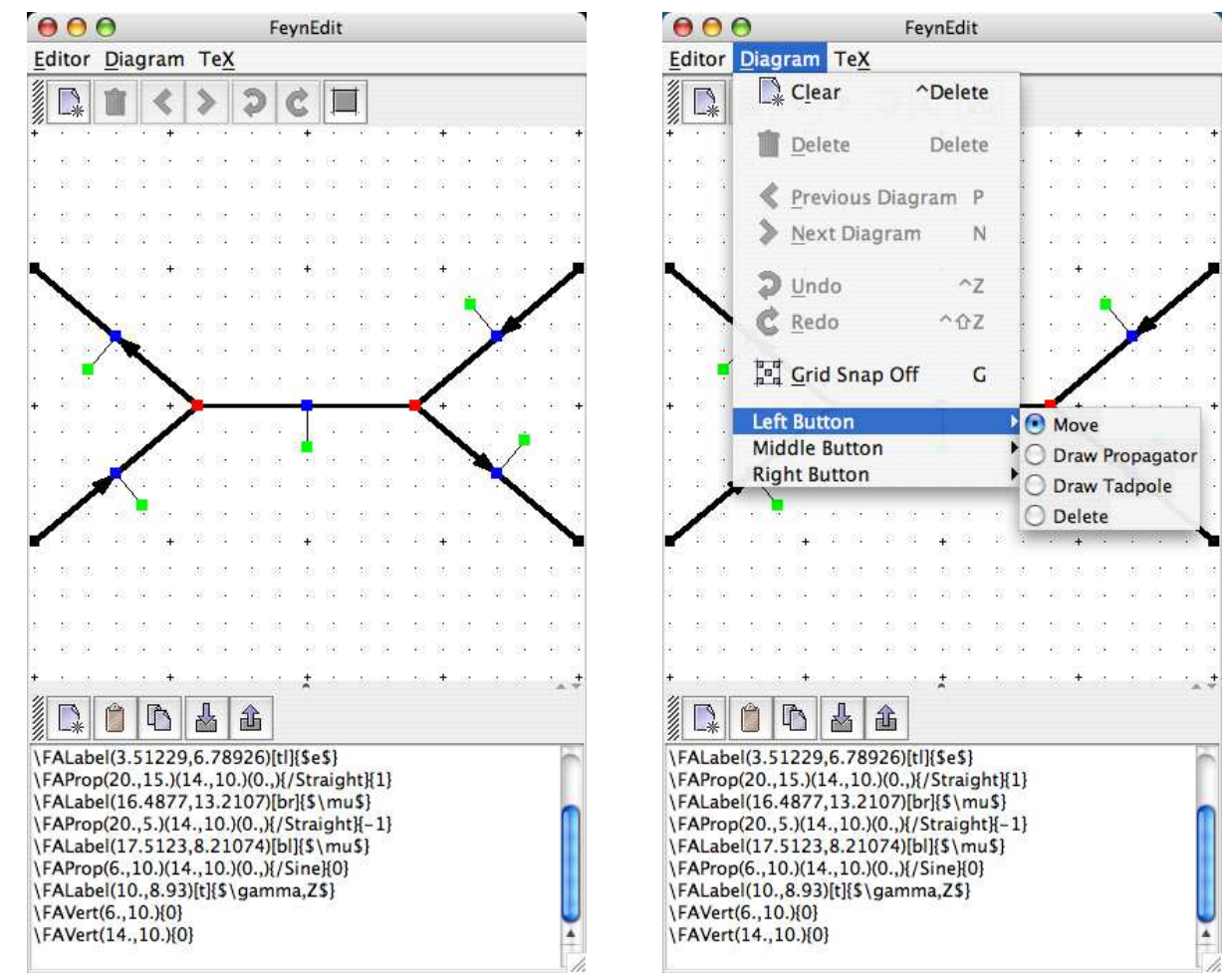

Figure 1: Left panel: The diagram pasted into FeynEdit and displayed. Right panel: The Mouse Button Assignment Menu in FeynEdit.

In the default setup, the left mouse button moves objects, the middle mouse button draws tadpoles, and the right mouse button draws propagators. The assignment of the mouse button can be changed in the Mouse Button Assignment menu (Fig. 1, right panel).

\section{Mathematica Interface}

The new Mathematica Interface turns the generated stand-alone Fortran code into a Mathematica function for evaluating the cross-section or decay rate as a function of user-selected model parameters. The benefits of such a function are obvious, as the whole instrumentarium of Mathematica commands can be applied to them. For example, one can simply use the Mathematica function ContourPlot to produce a contour plot of the cross-section.

Interfacing is done using the MathLink protocol. It is important to realize that the cross-section is not evaluated in Mathematica, but in Fortran, and only the numerical results computed by the Fortran code are transferred back to Mathematica.

\subsection{Input}

The changes necessary to produce a MathLink executable are by design minor and affect only the file run.F, where the user has to choose which model parameters are interfaced from Mathematica. A typical line in the stand-alone version of run.F might look like:

\#define LOOP1 do $1 \mathrm{~TB}=5,50,5$ 
This declares a loop over the variable TB ( $=\tan \beta$ in the MSSM model file) running from 5 to 50 in steps of 5. To turn this code into a Mathematica program, with TB appearing as an argument of the Mathematica function, the only modification necessary is to change the above line into

\#define LOOP1 call MmaGetReal(TB)

The variable TB is 'imported' from Mathematica now, i.e. the cross-section function in Mathematica becomes a function of TB hereby.

The user has full control over which variables are 'imported' from Mathematica and which are set in Fortran. Specifically, the invocations of MmaGetReal and its companion subroutine MmaGetComplex serve two purposes. At compile time they determine with which arguments the Mathematica function is generated, and at run time they actually transfer the function's arguments to the specified Fortran variables.

Once the makefile detects the presence of these subroutines, it automatically generates interfacing code and compiles a MathLink executable. For a file run.F the corresponding MathLink executable is also called run, as in the stand-alone case.

\subsection{Output}

Similar to the MmaGetReal invocations, the Fortran program can also 'export' variables to Mathematica. The line that prints a parameter in the stand-alone code is of the form

\#define PRINT1 SHOW "TB", TB

To transmit the value of TB to Mathematica, this becomes

\#define PRINT1 call MmaPutReal ("TB", TB)

\subsection{Usage}

Once the changes to run.F are made, the program run is compiled as usual:

./configure

make

It is then loaded in Mathematica with

Install ["run" ]

which makes a Mathematica function of the same name, run, available. There are two ways of invoking it which correspond closely to the command-line invocation of the stand-alone executable:

- Compute a differential cross-section at $\sqrt{s}=$ sqrt $\mathrm{S}$ :

$$
\text { run }[\text { sqrts, } \arg 1, \arg 2, \ldots]
$$

- Compute a total cross-section for sqrt Sfrom $\leqslant \sqrt{s} \leqslant$ sqrt Sto:

$$
\text { run }[\{\text { sqrtSfrom, sqrtSto }\}, \arg 1, \arg 2, \ldots]
$$

The extra arguments $\arg 1, \arg 2, \ldots$ are precisely the variables imported from the Fortran code, such as TB in the example above. 


\subsection{Data Retrieval}

Both the parameters exported from the Fortran code and the computed data (cross-section, decay rate, etc.) are transferred in sets, meaning that a separate Mathematica definition is made for each set. This has the important advantage that if the calculation is prematurely aborted, parameters and data transferred so far are still accessible. Such sets might look like

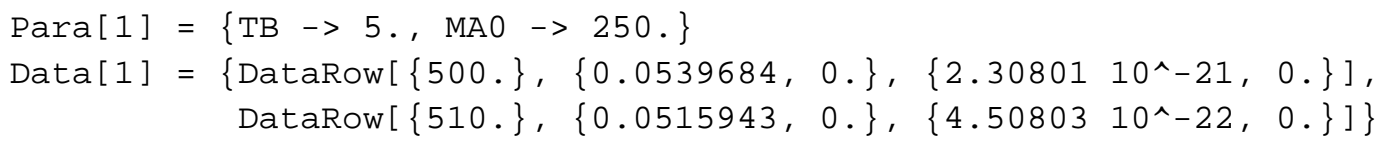

Not surprisingly, the actual return value of the function run is (only) an integer which indicates how many sets have been transferred.

The Para sets contain the parameters exported from the Fortran code. The Data sets contain for every data point computed a DataRow object which has three arguments:

- the unintegrated kinematical variables, here e.g. $\{500\}=.\{\sqrt{s}\}$,

- the tree-level cross-section and one-loop correction, here e.g. $\{0.0539684,0\}=.\left\{\sigma_{\text {tot }}^{\text {tree }}, \sigma_{\text {tot }}^{1-\text { loop }}\right\}$, and

- the integration errors of these quantities, here e.g. $\left\{2.3080110^{\wedge}-21,0.\right\}=\left\{\Delta \sigma_{\text {tot }}^{\text {tree }}, \Delta \sigma_{\text {tot }}^{1-\text { loop }}\right\}$.

\section{Splitting Abbreviations}

As a side effect of FormCalc's abbreviationing technique, the evaluation of the abbreviations constitute a major part of an amplitude calculation. Timings for a one-loop calculation may very roughly look like

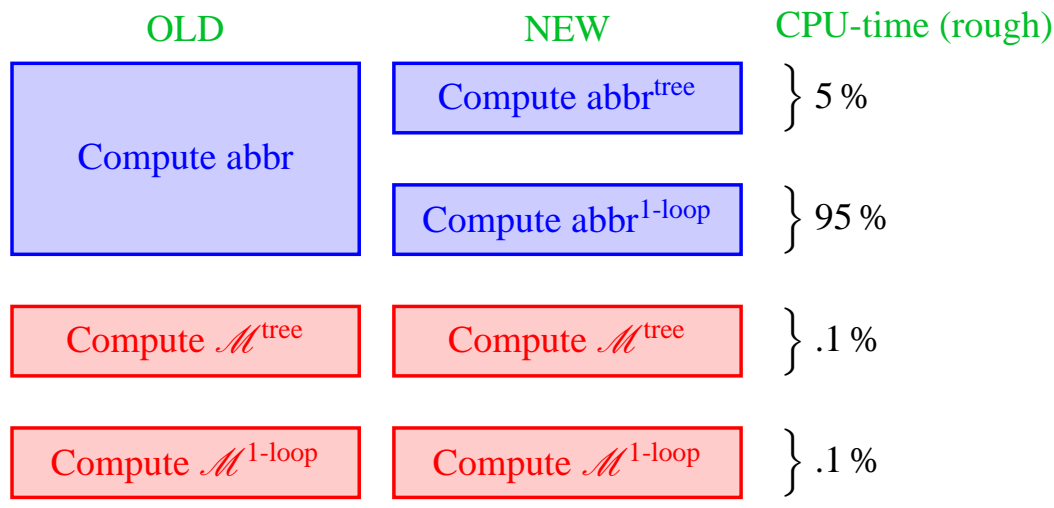

The old design had the obvious disadvantage that evaluating only the tree-level part would take about as much CPU time as the full one-loop amplitude. The current version thus splits the abbreviations into those that are needed for the tree-level part and the rest, and the main subroutine SquaredME correspondingly has an additional flag to choose whether to compute only the treelevel part.

The present set-up of the Fortran driver code does not (yet) make use of this splitting automatically. The most obvious application would be a separate phase-space integration of tree-level and one-loop component. 


\section{Fierz Identities in four dimensions}

The Fierz identities rearrange fermion chains by switching spinors, i.e.

$$
\left\langle 1\left|\Gamma_{i}\right| 2\right\rangle\left\langle 3\left|\Gamma_{j}\right| 4\right\rangle=\sum c_{k l}\left\langle 1\left|\Gamma_{k}\right| 4\right\rangle\left\langle 3\left|\Gamma_{l}\right| 2\right\rangle
$$

This is important in particular if one wants to extract certain predefined structures from the amplitude, most notably Wilson coefficients.

FormCalc so far automatically used the Fierz identities on 2-dimensional (Weyl) spinors, where the application is straightforward. The latest FormCalc version offers also the 4-dimensional variant through the new FermionOrder option for the CalcFeynAmp function. It is used as in

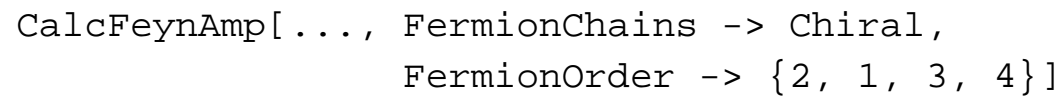

The FermionChains option chooses 4-dimensional (Dirac) spinors and the Fermionorder option instructs CalCFeynAmp to try to bring the spinor chains into the order $\langle 2|X| 1\rangle\langle 3|Y| 4\rangle$.

\section{Fully Analytic Amplitudes}

The 'smallest' object appearing in the output of CalcFeynAmp is a four-vector. The components, which reflect a particular phase-space parameterization, are inserted only later, usually in the numerical part.

So far, only the squared matrix element could be computed fully analytically. This method avoids an explicit phase-space parameterization but has the disadvantage that the size of the squared matrix element grows quadratically with the size of the amplitude. For example, for a fermionic amplitude $\mathscr{M}=\sum^{N} c_{i} F_{i}$, where the $F_{i}$ are (products of) fermion chains, the squared matrix element is computed as $|\mathscr{M}|^{2}=\sum^{N^{2}} c_{i} c_{j}^{*}\left(F_{i} F_{j}^{*}\right)$.

The new add-on package VecSet (loaded with $<<$ FormCalc 'tools 'VecSet ') makes two new functions available with which is is possible to express an amplitude in terms of phasespace parameters fully analytically:

- In a first step, the external vectors need to be set with the VecSet function. For example, VecSet $[1, \mathrm{~m} 1, \mathrm{p} 1,\{0,0,1\}]$ sets the momentum, polarization vectors, and spinors for external particle \#1 with mass $\mathrm{m} 1$ and three-momentum $\mathrm{p} 1$ moving in the direction $\{0,0,1\}$. This function is very similar to its Fortran namesake in the FormCalc drivers library.

- Once all external vectors have been set, an amplitude amp can be evaluated for example with ToComponents [amp, "+-+-"], where the "+-+-" are the polarizations of the external particles. ToComponents delivers an expression in terms of the phase-space parameters used in VecSet. 


\section{New Functions for Renormalization Constants}

New functions which simplify the definition of renormalization constants (RCs) have been introduced (the precise definitions of these quantities are listed in the FormCalc manual):

- MassRC $[f]$ - the mass $\mathrm{RC} \delta M_{f}$,

- MassRC $\left[f_{1}, f_{2}\right]$ - the mass RC $\delta M_{f_{1} f_{2}}$,

- FieldRC $[f]$ - the field RC $\delta Z_{f}$,

- FieldRC $\left[f_{1}, f_{2}\right]--$ the field $\mathrm{RC} \delta Z_{f_{1} f_{2}}$,

- TadpoleRC $[f]$ - the tadpole RC $\delta T_{f}$,

- WidthRC $[f]$ - the width $\Gamma_{f}$.

Using these function, the entire renormalization section of the Standard Model now fits on half a page (see FeynArts' SM.mod). The main aspect is to avoid mistakes, however: for example, a subtle index error in the fermion mixing counterterm in SM. mod has been discovered through the new RC functions.

\section{Separate Diagonalization Package}

The diagonalization routines included in FormCalc have been extended and made available as a separate package [3]. The following routines are available:

- HEigensystem diagonalizes a Hermitian matrix,

- SEigensystem diagonalizes a complex symmetric matrix,

- CEigensystem diagonalizes a general complex matrix,

- TakagiFactor computes the Takagi factorization of a symmetric matrix (e.g. the neutralino mass matrix),

- SVD performs the Singular Value Decomposition.

All routines are based on the Jacobi algorithm. This is conceptually simple but scales less favourably than e.g. the QR method. The applicability range is thus small to medium-size matrices. See also the timings in Fig. 2.

Use of the Jacobi algorithm results in rather compact code ( $\sim 3$ kBytes each), which is therefore easy to adapt to own conventions. The library is implemented in Fortran 77, but has a C/C++ and Mathematica interface. It stands under the LGPL license. 


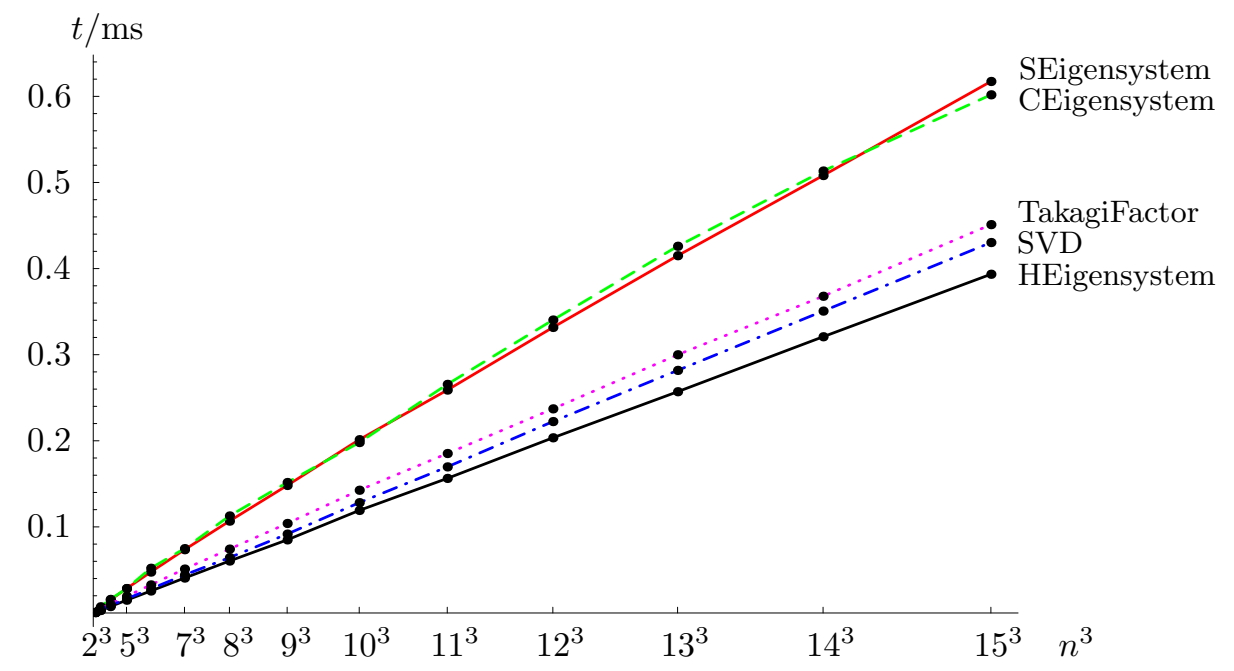

Figure 2: Timings of the diagonalization routines on an AMD X2-5000.

\section{Summary and Availability}

- The drawing tool FeynEdit is available from http://www.feynarts.de as an extra package (not part of FeynArts).

- The FormCalc version 5.3 contains the main new features

- Mathematica interface,

- Abbreviations are split into tree-level and loop parts,

- Fierz identitites in four dimensions are implemented,

- Fully analytic amplitudes are possible through an add-on package,

- New functions for renormalization constants

and is available from http://www.feynarts.de/formcalc.

- FormCalc's diagonalization routines have been extended and modeled into an own package which is available from http://www.feynarts.de/diag.

\section{References}

[1] T. Hahn, Comp. Phys. Commun. 140 (2001) 418 [hep-ph/0012260].

[2] T. Hahn, M. Pérez-Victoria, Comp. Phys. Commun. 118 (1999) 153 [hep-ph/9807565].

[3] T. Hahn, physics/0607103. 